\title{
FIRST VISUAL ORBIT FOR THE PROTOTYPICAL COLLIDING-WIND BINARY WR 140
}

\author{
J. D. Monnier ${ }^{1}$, Ming Zhao ${ }^{1}$, E. Pedretti ${ }^{1,2}$, R. Millan-Gabet ${ }^{3}$, J.-P. Berger ${ }^{4}$, W. Traub ${ }^{5}$, F. P. Schloerb ${ }^{6}$,

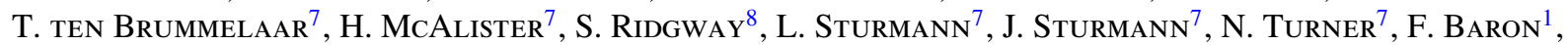 \\ S. Kraus ${ }^{1}$, A. TanNirkulam ${ }^{1,9}$, and P. M. Williams ${ }^{10}$ \\ ${ }^{1}$ University of Michigan Astronomy Department, 941 Dennison Bldg, Ann Arbor, MI 48109-1090, USA; monnier@umich.edu \\ ${ }^{2}$ European Organisation for Astronomical Research in the Southern Hemisphere, Karl Schwarzschild Strasse 2, 85748 Garching bei München, Germany \\ ${ }^{3}$ California Institute of Technology, NASA Exoplanet Science Institute, Pasadena, CA 91125, USA \\ ${ }^{4}$ IPAG, CNRs/UMR 5571, Universite J. Fourier, BP-53, F-38041 Grenoble Cedex, France \\ 5 Jet Propulsion Laboratory, California Institute of Technology, M/S 301-355, 4800 Oak Grove Drive, Pasadena, CA 91109, USA \\ ${ }^{6}$ Department of Astronomy, University of Massachusetts, Amherst, MA 01003-4610, USA \\ 7 The CHARA Array of Georgia State University, Mount Wilson, CA 91023, USA \\ ${ }^{8}$ National Optical Astronomy Observatory, 950 North Cherry Avenue, Tucson, AZ 85719, USA \\ ${ }^{9}$ Center for Micro Finance, The Institute for Financial Management and Research, IITM Research Park, A1, 10th floor, Taramani, Chennai 600113, India \\ ${ }^{10}$ Institute for Astronomy, University of Edinburgh, Royal Observatory, Blackford Hill, Edinburgh, UK \\ Received 2011 September 20; accepted 2011 October 11; published 2011 October 25
}

\begin{abstract}
Wolf-Rayet (WR) stars represent one of the final stages of massive stellar evolution. Relatively little is known about this short-lived phase and we currently lack reliable mass, distance, and binarity determinations for a representative sample. Here we report the first visual orbit for WR 140 (= HD193793), a WC7 + O5 binary system known for its periodic dust production episodes triggered by intense colliding winds near periastron passage. The Infrared-Optical Telescope Array and Center for High Angular Resolution Astronomy interferometers resolved the pair of stars in each year from 2003 to 2009, covering most of the highly eccentric, 7.9 year orbit. Combining our results with the recently improved double-line spectroscopic orbit of Fahed et al., we find the WR 140 system is located at a distance of $1.67 \pm 0.03 \mathrm{kpc}$, composed of a WR star with $M_{\mathrm{WR}}=14.9 \pm 0.5 M_{\odot}$ and an $\mathrm{O}$ star with $M_{\mathrm{O}}=35.9 \pm 1.3 M_{\odot}$. Our precision orbit yields key parameters with uncertainties $\sim 6 \times$ smaller than previous work and paves the way for detailed modeling of the system. Our newly measured flux ratios at the near-infrared $H$ and $K s$ bands allow a spectral energy distribution decomposition and analysis of the component evolutionary states.
\end{abstract}

Key words: binaries: visual - infrared: stars - stars: individual (WR 140, HD 193793) - stars: Wolf-Rayet techniques: interferometric

\section{INTRODUCTION}

Wolf-Rayet (WR) stars are evolved massive stars characterized by intense mass loss through radiation-driven winds. These hot, emission-line stars are especially helium-rich, having lost most of their hydrogen envelope through winds or interaction with a companion. The range of progenitors that become WR stars is not well understood and establishing a massive star evolution sequence represents one of the most serious challenges for modern stellar theory (see recent review by Crowther 2007).

Concrete mass and distance determinations are crucial to making further progress, but this is difficult due to the large distances from Earth at which most WR stars lie. According to van der Hucht (2001), there are only 19 WR stars with mass estimates based on spectroscopic orbits and the vast majority of these are for short-period systems with periods between 1 and 100 days. Longer period binaries are less likely to have had interactions between the components but are difficult to characterize due to the lower orbital speeds.

The subject of this Letter is WR 140 (= HD 193793), a WR binary system with a 7.9 year period. Williams et al. (1987) first noticed a mysterious episodic infrared variability and follow-up observations (Williams et al. 1990; Moffat et al. 1987) established the cause to be dust creation near periastron of a highly eccentric orbit, likely catalyzed in the collidingwind interface between the WR star and O-star winds (Usov 1991). Since this time, WR 140 (WC7 + O5) has been subject to many monitoring campaigns, including infrared (Williams et al. 2009), radio (White \& Becker 1995), and in radial velocity
(Fahed et al. 2011). Recently, progress toward a proper visual orbit was made by the single-epoch detection of the binary using the Infrared-Optical Telescope Array (IOTA) interferometer (Monnier et al. 2004) and through repeated imaging of the rotating colliding-wind region using the Very Long Baseline Array (VLBA; Dougherty et al. 2005). Despite the wealth of data, mass estimates have suffered from large errors $(\sim 20 \%)$ due to lack of a high-quality visual orbit to go along with precise spectroscopic data.

In this Letter, we report seven epochs of binary observations at the near-infrared $H$ and $K s$ bands with the IOTA and Center for High Angular Resolution Astronomy (CHARA) interferometers. Our data span seven years, allowing us to construct the first complete visual orbit for WR 140. We combine this with recent spectroscopic work to determine precise masses and orbital parallax.

\section{OBSERVATIONS}

\subsection{IOTA}

Observations of WR 140 in 2003, 2004, and 2005 were obtained with the IOTA interferometer (Traub et al. 2003). IOTA was located on Mt. Hopkins (Arizona) and consisted of three $0.45 \mathrm{~m}$ telescopes that were movable among 17 stations along two orthogonal linear arms (telescopes $\mathrm{A}$ and $\mathrm{C}$ could move along the $35 \mathrm{~m}$ northeastern arm, while telescope B could move along the $15 \mathrm{~m}$ southeastern arm). By observing a target in many different array configurations, IOTA could synthesize an aperture of $35 \mathrm{~m} \times 15 \mathrm{~m}$ (corresponding to an angular resolution 
Table 1

Observing Log for WR 140

\begin{tabular}{|c|c|c|c|c|}
\hline $\begin{array}{l}\text { Orbital } \\
\text { Phase }^{\mathrm{a}}\end{array}$ & $\begin{array}{l}\text { Date } \\
\text { (UT) }\end{array}$ & $\begin{array}{l}\text { Interferometer } \\
\text { (Configuration) }\end{array}$ & $\begin{array}{c}\lambda_{0} \\
(\mu \mathrm{m})\end{array}$ & $\begin{array}{l}\text { Bandwidth } \\
(\mu \mathrm{m})\end{array}$ \\
\hline 2.296 & 2003 Jun 17 & IOTA $^{b}(\mathrm{~A} 35 \mathrm{C} 15 \mathrm{C} 10)$ & 1.650 & 0.248 \\
\hline 2.406 & 2004 Apr 30 & IOTA (A35B15C10) & 1.650 & 0.248 \\
\hline 2.407 & 2004 May 1 & IOTA (A35B15C10) & 1.650 & 0.248 \\
\hline 2.417 & 2004 May 30 & IOTA (A35B15C10) & 1.650 & 0.248 \\
\hline 2.417 & 2004 Jun 1 & IOTA (A35B15C10) & 1.650 & 0.248 \\
\hline 2.418 & 2004 Jun 4 & IOTA (A35B15C10) & 1.650 & 0.248 \\
\hline 2.419 & 2004 Jun 5 & IOTA (A35B15C10) & 1.650 & 0.248 \\
\hline 2.419 & 2004 Jun 6 & IOTA (A35B15C10) & 1.650 & 0.248 \\
\hline 2.547 & 2005 Jun 11 & IOTA (A35B15C10) & 1.650 & 0.248 \\
\hline 2.547 & 2005 Jun 13 & IOTA (A35B15C10) & 1.650 & 0.248 \\
\hline 2.548 & 2005 Jun 14 & IOTA (A35B15C10) & 1.650 & 0.248 \\
\hline 2.548 & 2005 Jun 15 & IOTA (A35B15C10) & 1.650 & 0.248 \\
\hline 2.548 & 2005 Jun 16 & IOTA (A35B15C10) & 1.650 & 0.248 \\
\hline 2.549 & 2005 Jun 17 & IOTA (A35B15C10) & 1.650 & 0.248 \\
\hline 2.549 & 2005 Jun 18 & IOTA (A35B15C10) & 1.650 & 0.248 \\
\hline 2.698 & 2006 Aug 22 & CHARA $^{\mathrm{c}}(\mathrm{W} 2-\mathrm{E} 2)$ & 2.133 & 0.350 \\
\hline 2.799 & 2007 Jun 11 & CHARA (W2-E2) & 1.673 & 0.274 \\
\hline 2.800 & 2007 Jun 13 & CHARA (W2-E2) & 2.133 & 0.350 \\
\hline 2.926 & 2008 Jun 14 & CHARA (W2-E2) & 1.673 & 0.274 \\
\hline 2.927 & 2008 Jun 16 & CHARA (W2-S1) & 2.133 & 0.350 \\
\hline 2.927 & 2008 Jun 17 & CHARA (W1-S2) & 2.133 & 0.350 \\
\hline 2.928 & 2008 Jun 18 & CHARA (W1-S2) & 2.133 & 0.350 \\
\hline 3.054 & 2009 Jun 20 & CHARA (W1-S2) & 2.133 & 0.350 \\
\hline 3.055 & 2009 Jun 22 & CHARA (W1-S2) & 2.133 & 0.350 \\
\hline 3.055 & 2009 Jun 23 & CHARA (S2-E2) & 2.133 & 0.350 \\
\hline 3.056 & 2009 Jun 25 & CHARA (S2-E2) & 2.133 & 0.350 \\
\hline
\end{tabular}

Notes. All calibrated OI-FITS data available upon request.

a Orbital phase assuming $T_{0}=2446156.2$ (MJD), $P=2896.5$ days (Fahed et al. 2011).

b IOTA calibration employed the following calibrators (all sizes were estimated using getCal): HD 192985 (0.46 \pm 0.06 mas), HD 193631 (0.31 \pm 0.28 mas), HD 126035 (0.78 \pm 0.24 mas), HD 193664 (0.58 \pm 0.05 mas), HD 193961 (0.24 \pm 0.06 mas).

c CHARA calibration employed the following calibrators (all sizes estimated using getCal, except HD196360): HD 192985 (0.46 \pm 0.06 mas), HD 193631 (0.31 \pm 0.28 mas), HD 196360 (0.61 \pm 0.05 mas), HD 192640 (0.40 \pm 0.15 mas $)$, HD 195194 (0.63 \pm 0.13 mas $)$.

of $(\lambda / B) \sim 5 \times 12$ mas at $1.65 \mu \mathrm{m})$. The observations of WR 140 from 2003 were first reported in Monnier et al. (2004).

All of the IOTA observations included three simultaneous baselines using the broadband $H$ filter and the light beams from the three telescopes were interfered using the single-mode IONIC3 combiner (Berger et al. 2003). Basic data reduction procedures were the same as described in several previous IOTA papers (e.g., Monnier et al. 2006) and final error estimation followed the study of the $\lambda$ Vir binary by Zhao et al. (2007); we applied $2 \%$ relative and $\Delta \mathcal{V}^{2}=0.02$ additive systematic errors to all our measured visibility amplitude $\mathcal{V}^{2}$ data. We found our closure phases were partially corrupted by bandwidth smearing effects and we only used $\mathcal{V}^{2}$ data for our orbit fitting (see detailed description and simulation of these effects explored by Zhao et al. 2007). Table 1 contains a detailed log of the IOTA observations.

\subsection{CHARA}

Observations of WR 140 in the years 2006, 2007, 2008, and 2009 were obtained with the CHARA Array. CHARA is located on Mt. Wilson (California) and consists of six fixed $1 \mathrm{~m}$ aperture telescopes with baselines ranging from 30 to $330 \mathrm{~m}$. CHARA is currently the longest baseline optical interferometer in the world and can reach angular resolutions of $\sim 0.5$ mas in the near-infrared. The CHARA facility and the two-beam CLASSIC combiner used in this work are described by ten Brummelaar et al. (2005).

Most of the CHARA observations were carried out in the $K s$ band although a few data points were taken in the $H$ band. The observing dates, wavelengths, and baselines can be found in Table 1. We reduced the data using an IDL-based suite of routines written by one of us (J.D.M.) and these have been previously described in Tannirkulam et al. (2008). For this Letter, we adopted $10 \%$ relative and $\Delta \mathcal{V}^{2}=0.02$ additive errors to account for systematic calibration errors. ${ }^{11}$

We have reported all the calibrators and adopted sizes in Table 1. Uniform disk (UD) diameters of interferometer calibrators were generally estimated using getCal, a spectral energy distribution (SED) fitting routine maintained and distributed by the NASA Exoplanet Science Institute (http://nexsci.caltech.edu), or SearchCal (Bonneau et al. 2006), a surface brightness algorithm maintained and distributed by the J. M. Mariotti Center (JMMC, http://jmmc.fr). We note that getCal does not produce as accurate diameter estimates as searchCal in general, but getCal can be employed on a wider variety of calibrator spectral types and was more useful in this current Letter.

The final IOTA and CHARA calibrated $\mathcal{V}^{2}$ data were saved in OI-FITS format (Pauls et al. 2005) and all files are available upon request.

\section{ANALYSIS}

We have interpreted our $\mathcal{V}^{2}$ data using a binary model. Here we describe the components of the model and our fitting results.

\subsection{Description of the Binary Model}

Our model for WR 140 consists of two stars, each modeled as a UD. The size of the $\mathrm{O}$ star can be estimated based on the effective temperature and infrared flux estimate, while the WR star size is affected by the optically thick wind (we followed a similar procedure to Millour et al. 2007, to account for wind opacity). We found that both stars are much smaller than the resolution of CHARA and so our final results are not sensitive to our adopted UD size of 0.05 mas for the WR star and 0.07 mas for the $\mathrm{O}$ star.

The WR/O-star flux ratios at the $H$ and $K s$ bands were separately fitted but held constant across all epochs. The nearinfrared (NIR) flux monitoring by Taranova \& Shenavrin (2011) showed that $52 \%$ of the $K$-band emission during the 2009 epoch was from the outburst dust shell and was assumed to be overresolved by CHARA. No other epochs were affected by dust emission. For a given set of orbital elements $(a, e, i, \omega, \Omega$, $P, T_{0}$ ), we can predict the separation and position angle at the time of each observation. The apparent brightness ratio can be affected by the finite bandwidth of the observations ("bandwidth-smearing") and we have accounted for the square bandpasses of the $H$ and $K s$ filters using the basic procedure described in Zhao et al. (2007). The bandwidth-smearing correction was insignificant for the short baseline IOTA data, but did affect the longer baseline CHARA data at the 5\%-10\% level.

\footnotetext{
11 The calibration errors were lower for IOTA-IONIC3 because of the use of single-mode fibers while CHARA-CLASSIC is a "free-space combiner" with only limited spatial filtering.
} 


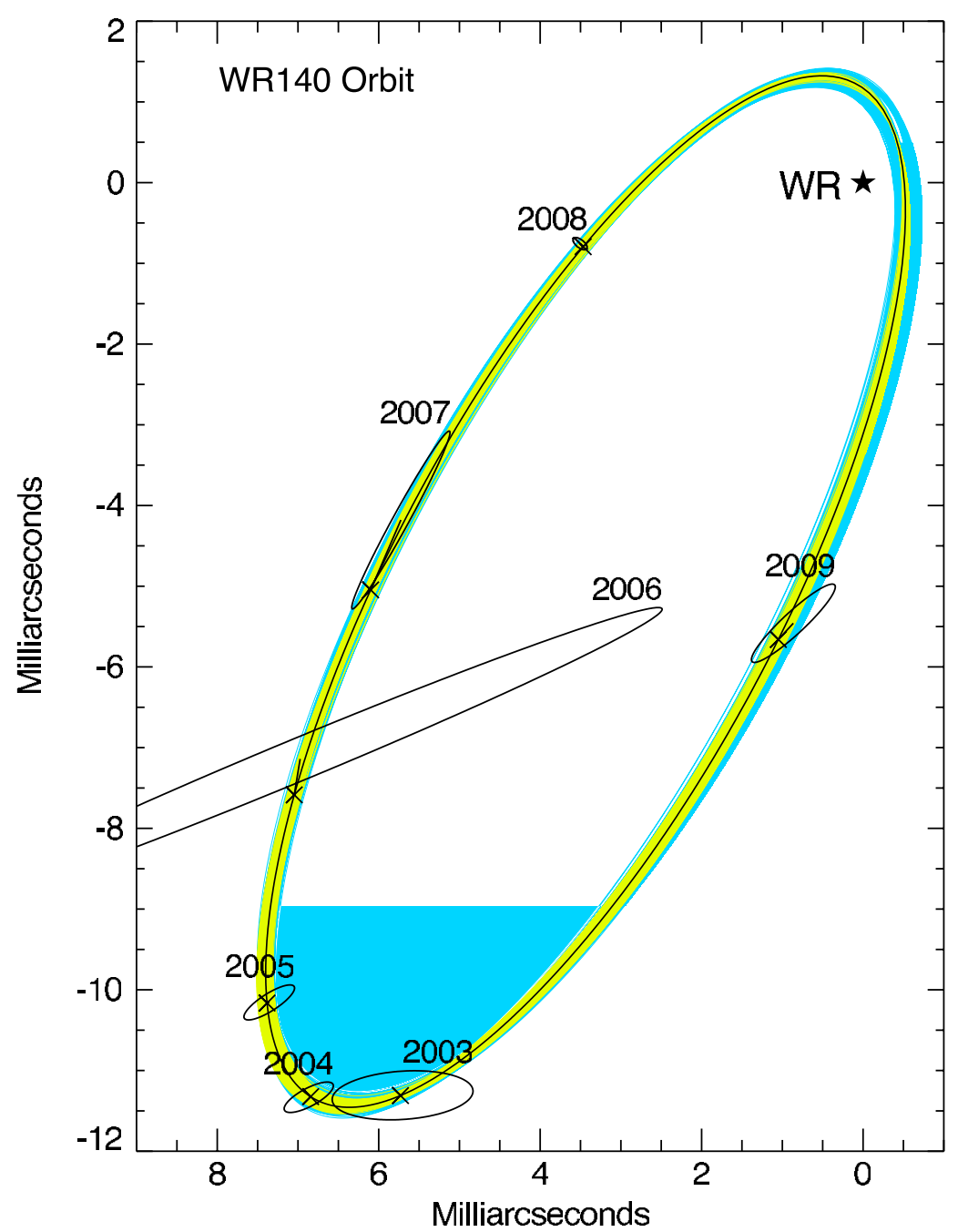

Figure 1. $3 \sigma$ band of allowed orbits based on our visual data alone (thick blue band) and for the more-constraining, joint visual/spectroscopic solution (yellow band). The best-fit joint solution is shown with solid line. For each year, the data were analyzed separately and the positions of the O star with respect to WR star are shown here, marked by error ellipses (see Table 2). The best-fit orbit prediction for each epoch is connected to each corresponding error ellipse.

For visualization purposes, we also collected each year's data and fitted for relative positions. The best-fitting locations of the O star relative to the WR star for each observation year has been included in Figure 1 and allows for comparison with our final orbit fitting results. The size and shape of each epoch's allowed region (an error ellipse containing the $68 \%$ confidence region) vary significantly from year to year due to differences in the quantity and quality of the $\mathcal{V}^{2}$ data. We also included the parameters of these best-fit locations in Table 2.

To validate our choice of this simple binary model, we also carried out model-independent image reconstructions for the 2003-2005 IOTA/IONIC3 data. We confirmed that the system is dominated by two point sources. We were interested to see if there could be any sign of the colliding-wind zone between the stars, but no extra emission was seen in this region (at the level of a few percent of the peak emission). The brightest emission not coming from the two stars showed up to the northeast of the system in some epochs at the $2 \%-5 \%$ level-likely an artifact from residual miscalibration.

\subsection{Orbit Fitting Procedure}

To arrive at our final orbital solutions, we fit directly to the $\mathcal{V}^{2}$ values. We carried out two different fitting exercises that differed in how we incorporated spectroscopic data.
First, we wanted to carry out an orbital fit as independent as possible from the recent spectroscopic orbit of Fahed et al. (2011). This allows us to independently confirm the crucial orbital elements $e$ and $\omega$, although we adopted their values for the period $(P)$ and time of periastron $\left(T_{0}\right)$ in this procedure. The best-fitting orbital elements (reduced $\chi^{2}=0.52$ ) are compiled in the right column of Table 3. Error bars were estimated using 1000 bootstrap resamplings (Efron \& Tibshirani 1993) of the data (grouped by night). This is the same procedure recently applied for the visual orbit of $\alpha$ Oph (Hinkley et al. 2011). In order to capture the uncertainties in the Fahed et al. (2011) estimates for $P$ and $T_{0}$, we did not strictly fix these quantities during the bootstrap fits but rather used Monte Carlo sampling based on the Fahed et al. (2011) uncertainty estimates.

The IOTA + CHARA visual orbit compares favorably with the spectroscopically determined orbital elements from Fahed et al. (2011).

1. $e: 0.8962 \pm 0.0014$ (Fahed's orbit "This paper+M03") compared to eccentricity $0.901_{-.004}^{+.006}$ (this work alone). These values are compatible and confirm the high orbit eccentricity.

2. $\omega: 44.6 \pm 1.1$ (Fahed's orbit "This orbit+M03") compared to $48.2 \pm 1.3$ (this work alone). This is slightly discrepant, although note that one of the orbital solutions presented in 
Table 2

Position of O star with Respect to WR Star

\begin{tabular}{|c|c|c|c|c|c|}
\hline \multirow{2}{*}{$\begin{array}{l}\text { Mean Date } \\
\text { (UT) }\end{array}$} & \multicolumn{2}{|c|}{ Relative Position } & \multicolumn{3}{|c|}{ Error Ellipse $^{\mathrm{a}}$} \\
\hline & $\begin{array}{c}\text { East } \\
\text { (mas) }\end{array}$ & $\begin{array}{l}\text { North } \\
\text { (mas) }\end{array}$ & $\begin{array}{l}\text { Major } \\
\text { (mas) }\end{array}$ & $\begin{array}{l}\text { Minor } \\
\text { (mas) }\end{array}$ & $\begin{array}{l}\text { P.A. Major } \\
(\mathrm{E} \text { of } \mathrm{N})\end{array}$ \\
\hline 2003 Jun 17 & 5.70 & -11.31 & 0.87 & 0.30 & -86 \\
\hline 2004 May 25 & 6.87 & -11.33 & 0.34 & 0.11 & -61 \\
\hline 2005 Jun 15 & 7.36 & -10.16 & 0.37 & 0.11 & -57 \\
\hline 2006 Aug 22 & 6.97 & -7.14 & 4.85 & 0.26 & 112 \\
\hline 2007 Jun 12 & 5.73 & -4.18 & 1.25 & 0.10 & -28 \\
\hline 2008 Jun 17 & 3.51 & -0.76 & 0.11 & 0.05 & 51 \\
\hline 2009 Jun 23 & 0.86 & -5.46 & 0.70 & 0.13 & -47 \\
\hline
\end{tabular}

Note.

a Error ellipse contains $68 \%$ confidence interval and is specified by the \pm error in the two orthogonal directions (in milliarcseconds) specified by the position angle of the ellipse major axis (degrees east of north). See Figure 1 for graphical representation.

Table 3

Orbital Parameters for WR 140 (Adopting WR as Primary Star, O as Secondary Star)

\begin{tabular}{|c|c|c|}
\hline Parameter & $\begin{array}{l}\text { This Work with } \\
\text { Fahed et al. (2011) Prior }\end{array}$ & This Work alone ${ }^{\mathrm{a}}$ \\
\hline Flux ratio $^{\mathrm{b}}(H$ band $)$ & \multicolumn{2}{|c|}{$1.37 \pm 0.03$} \\
\hline Flux ratio ( $K s$ band) & \multicolumn{2}{|c|}{$1.94 \pm 0.06$} \\
\hline Semi-major axis (mas) & $8.82 \pm 0.05$ & $8.99_{-0.22}^{+0.16}$ \\
\hline Eccentricity & $0.8964_{-0.0007}^{+0.0004}$ & $0.901_{-.004}^{+.006}$ \\
\hline Inclination (deg) & $119.6 \pm 0.5$ & $118.9_{-0.5}^{+1.3}$ \\
\hline$\omega(\operatorname{deg})$ & $46.8 \pm 0.4$ & $48.2 \pm 1.9$ \\
\hline$\Omega(\operatorname{deg})$ & $353.6 \pm 0.4$ & $354.2_{-0.5}^{+0.9}$ \\
\hline Period (days) & $2896.35 \pm 0.20$ & $\left(2896.5_{-1.5}^{+0.2}\right)$ \\
\hline$T_{0}(\mathrm{MJD})$ & $46154.8 \pm 0.8$ & $\left(46155.7_{-3.3}^{+2.6}\right)$ \\
\hline$\sum \chi^{2} / \mathrm{dof}$ & 0.51 & 0.52 \\
\hline \multicolumn{3}{|c|}{ Derived physical quantities $^{\mathrm{c}}$} \\
\hline Distance (kpc) & $1.67 \pm 0.03$ & $1.60_{-0.07}^{+0.11}$ \\
\hline$M_{\mathrm{WR}}\left(M_{\odot}\right)$ & $14.9 \pm 0.5$ & $13.9_{-1.2}^{+1.9}$ \\
\hline$M_{\mathrm{O}}\left(M_{\odot}\right)$ & $35.9 \pm 1.3$ & $33.1_{-2.8}^{+4.5}$ \\
\hline
\end{tabular}

Notes.

a All orbital elements were fitted to the visual orbit date presented here, except for the $P$ and $T_{0}$, which were adopted from Fahed et al. (2011).

${ }^{\mathrm{b}}$ Flux ratio is WR flux density/O5 flux density.

${ }^{c}$ The visual orbit above and the spectroscopic data from Fahed et al. (2011) were combined to derive the orbital parallax and relative masses. We emphasize that the high precision on the mass and distance requires both the visual orbit presented here and high-quality spectroscopic data of Fahed et al. (2011).

the Fahed et al. (2011) reports $\omega=47.5$ (Fahed's orbit "This paper;" middle solution in Table 2).

Much tighter constraints on the orbital elements can be attained by using the spectroscopic values from Fahed et al. (2011) as a prior in the visual orbit fit (the orbit labeled "This paper+M03"). The most important effect of this is to constrain the eccentricity, which is better determined by the Fahed et al. (2011) data set that sampled the periastron period very densely. Our visual observations missed the fast-changing orbital motion in early 2009 and thus cannot be expected to optimally constrain eccentricity.

The first column of Table 3 contains our best-fitting solution from the joint analysis (reduced $\chi^{2}=0.51$ ). We used the same bootstrap procedure to determine the error bars on each parameter. To visualize the range of orbits allowed by our solutions, we plotted all 1000 bootstrap orbits in Figure 1. As expected, the joint solution is better constraining, especially near periastron.

The last step in our analysis was to take the new orbital elements and use the spectroscopic $K$ values from Fahed et al. (2011) to calculate masses and orbital parallax. Strictly speaking, since the $K$ values depend slightly on the orbital elements themselves, we re-fitted the $\gamma$ and $K$ values for our orbital solutions using the radial velocity data in Fahed et al. (2011). This refinement is slightly more accurate than simply adopting the spectroscopic $a \sin i$ along with astrometry to derive inclination and distance. The final results for masses and distance are also included in Table 3.

Another way to view the remarkable quality of the fit is presented in Figure 2. Here we show the observed $\mathcal{V}^{2}$ as a function of the projected separation of the two components (joint orbital solution). This figure also shows the effect of bandwidth smearing for large projected separations. 


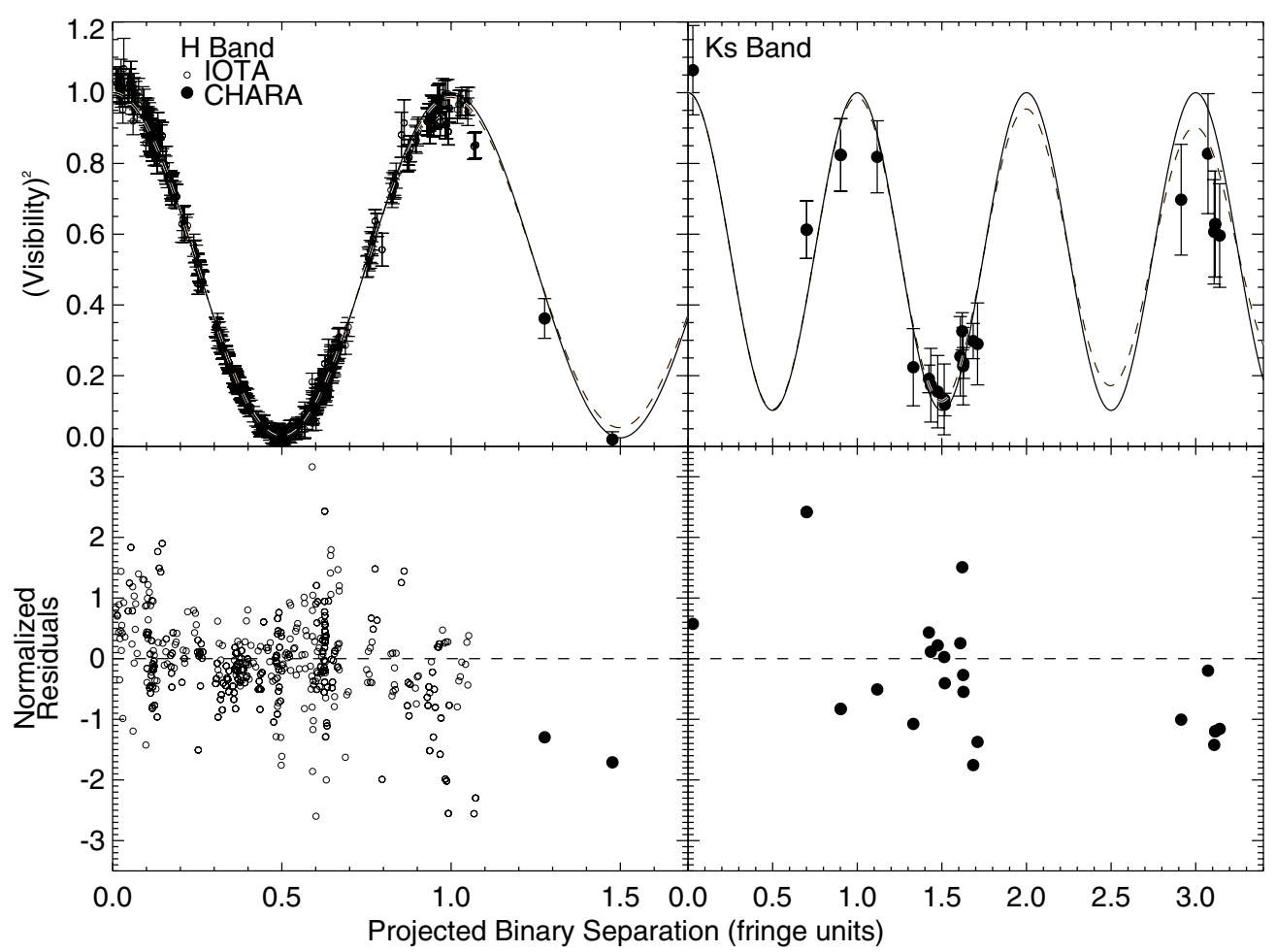

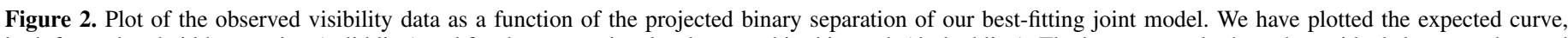

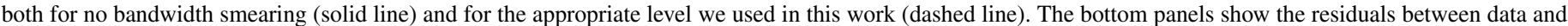

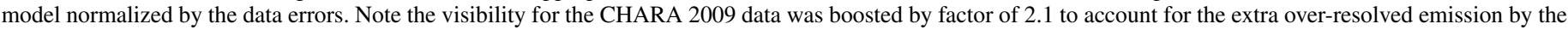
short-lived dust shell created at periastron (48\% stellar emission, 52\% dust; Taranova \& Shenavrin 2011).

\section{DISCUSSION AND FUTURE WORK}

The WR and O-star masses found here are similar to previous estimates (e.g., van der Hucht 2001) but with $\sim 6 \times$ smaller error bars $(\sim 3 \%-4 \%)$. As has been seen in other WC binary systems, we find that the WR star mass is less than $20 M_{\odot}$ and less than half the current mass of the O-star companion. These two qualities fit the interesting trend seen in the (six) WC stars with mass estimates (Crowther 2007). Our accurate distance will help to place these two stars in the Hertzsprung-Russel diagram for a stringent test of stellar evolution models that include mass loss. This is only the second galactic WR star to have a distance measured through (orbital) parallax (the other is $\gamma$ Vel; Millour et al. 2007). Note that the periastron distance of the orbit is $1.53 \mathrm{AU}$, which means the O-star companion is too distant to have affected the stellar evolution of the WR star-unless the WR star progenitor experienced a red supergiant stage (see case of WR 104; Tuthill et al. 2008) or the orbit has drastically changed due to mass loss.

The interferometry data allow us to measure the flux ratios of the two stars $\left(\right.$ Flux $\left._{(\mathrm{WR})} / \mathrm{Flux}_{(\mathrm{O})}\right)$ in the $H$ and $K s$ bands for the first time. Our $H$-band IOTA data employed closure phases, allowing us to identify the northwest component to be brightest in 2003. We have had to assume that this component is also brighter at the $K$ band since we lack closure phase data to break the $180^{\circ}$ degeneracy of single-baseline data.

Based on our flux ratios, the IR-bright component has a significantly redder color than the IR-faint component, consistent with the IR-bright component being the WR star. The shape of the non-thermal radio emission (Dougherty et al. 2005) also identifies the northwest (IR-bright) component in 2003 to be the WR star that is expected to possess the higher-momentum wind.
Armed with this knowledge, we can decompose the combined SED into their component SEDs. For the combined system, we adopt (non-dusty) $B V J H K$ magnitudes = $(7.28,6.89,5.71,5.35,5.02) \quad$ (Reed 2003; Taranova \& Shenavrin 2011) and assume the O5III star has colors given by Martins \& Plez (2006). The last ingredient we need is the least certain: the interstellar reddening. We will adopt the reddening law of Mathis (1990) with $R_{V}=3.1$ and with $A_{V}$ spanning $A_{V}=2.95$ from Morris et al. (1993) using the $2175 \AA$ feature and $A_{V}=2.06$ from SED colors (Conti \& Vacca 1990) ${ }^{12}$-as you will see, the large uncertainty in $A_{V}$ leads to large errors in our system luminosity despite our new well-constrained distance. We can now fix the $H$-band ratio to 1.37 from our IOTA data and solve for the remaining flux ratios (modulo the $A_{V}$ uncertainty). For $A_{V}=2.95$, the flux ratios at $B V J H K$ become $(2.46,1.64,1.05,1.37,1.93)$; for $A_{V}=2.06$ the flux ratios become $(0.37,0.35,0.88,1.37,2.09)$. The observed flux ratio at the $K s$ band $(1.94 \pm 0.06)$ is within the range seen here and is in good agreement with the high- $A_{V}$ case. Depending on which lines are used and which templates are adopted, Fahed et al. (2011) argue that the $\mathrm{O}$ star might range from $\sim 0.5$ to $\sim 3 \times$ brighter than the WR star in the optical band continuum-a wide range compatible with our derived flux ratios. Our prediction for $V$-band flux ratio varies by a factor of five (!) because of $A_{V}$ uncertainties and thus an interferometric measurement here could be exploited to strongly constrain the true reddening, a crucial component to luminosity determination which we now explore.

Using our new distance $(d=1.67 \mathrm{kpc})$ and the range of flux ratios, we find $M_{V}$ for the $\mathrm{O}$ star ranges from -6.11 to -5.94 and the $M_{V}$ for the WR star ranges from -6.6 to -4.8 . This would

\footnotetext{
${ }^{12}$ Here, we have used the relation that $A_{v}=1.1 A_{V}$ (e.g., Smith 1968).
} 
classify the $\mathrm{O} 5$ star as intermediate between giant and supergiant (cf. Martins \& Plez 2006). The WR star luminosity class is hardly constrained but the high- $A_{V}$ case yields unrealistically high luminosities as judged by other WC7 stars with known distances (via cluster membership; van der Hucht 2001). In an attempt to reconcile the differences, we scaled the fluxes for a $70 \mathrm{kK}$ CMFGEN model for the WC7 star from Smith et al. (2002) to match the measured flux ratio of 1.37 in the $H$ Band using a model SED for the O5 star from Martins et al. (2005). The WR/O5 flux ratios in $V$ and $v$ were found to be 1.05 and 0.73 , respectively, that in $V$ being affected by strong emission lines. The absolute magnitudes derived for the $05 \mathrm{star}, M_{V}=$ -6.37 and -5.65 for high- and low-reddening extremes, are near or above those for supergiants, whereas those for the WC star, $M_{v}=-6.7$ and -5.9 are both anomalously high. Resolution of the uncertain interstellar reddening of WR 140 is beyond the scope of the present Letter but is urgently required to exploit the determination of its parallax.

We expect our success here will motivate future observations of galactic WR stars using today's interferometers from visible to NIR wavelengths. The multi-wavelength flux ratios can help yield crucially needed new estimates of interstellar reddening, and the possibility to combine spectroscopic orbits with new interferometric visual orbits will allow accurate distance, mass, and luminosity measurements for a substantially larger set of galactic WR stars. Such a data set is important to test the current massive stellar evolution paradigm that tells us how main-sequence $\mathrm{O}$ stars move through the various stages of red supergiant, WR (WN and WC), and luminous blue variable before ultimately becoming a supernova.

We have appreciated discussions with Tony Moffat, Peter Tuthill, Debra Wallace, Bill Danchi, Sean Dougherty, and Remi Fahed during the (long) course of this work. We thank SAO, U. Mass, NSF AST-0138303, NSF AST-0352723, and NASA NNG05G1180G for supporting IOTA development and operations. We also acknowledge funding from GSU, the Keck Foundation, and NSF AST-0908253 for the CHARA Array. IONIC-3 was developed by LAOG (now IPAG) and LETI in the context of the IONIC collaboration (LAOG, IMEP, LETI), funded by the CNRS and CNES (France). Lastly we thank NSF AST-0807577 for support of University of Michigan researchers in this work. E.P. received funding from a Michelson Postdoctoral Fellowship and a Scottish Universities Physics
Alliance (SUPA) advanced fellowship. P.M.W. is grateful to the Institute for Astronomy for hospitality and continued access to the facilities of the Royal Observatory, Edinburgh. This research has made use of the SIMBAD database, operated at CDS, Strasbourg, France, and NASA's Astrophysics Data System (ADS) Bibliographic Services.

Facility: IOTA (IONIC3), CHARA (CLASSIC)

\section{REFERENCES}

Berger, J., Haguenauer, P., Kern, P. Y., et al. 2003, Proc. SPIE, 4838, 1099 Bonneau, D., Clausse, J.-M., Delfosse, X., et al. 2006, A\&A, 456, 789 Conti, P. S., \& Vacca, W. D. 1990, AJ, 100, 431

Crowther, P. A. 2007, ARA\&A, 45, 177

Dougherty, S. M., Beasley, A. J., Claussen, M. J., Zauderer, B. A., \& Bolingbroke, N. J. 2005, ApJ, 623, 447

Efron, B., \& Tibshirani, R. J. 1993, An Introduction to the Bootstrap (New York: Chapman and Hall)

Fahed, R., Moffat, A. F. J., Zorec, J., et al. 2011, MNRAS, in press

Hinkley, S., Monnier, J. D., Oppenheimer, B. R., et al. 2011, ApJ, 726, 104

Martins, F., \& Plez, B. 2006, A\&A, 457, 637

Martins, F., Schaerer, D., \& Hillier, D. J. 2005, A\&A, 436, 1049

Mathis, J. S. 1990, ARA\&A, 28, 37

Millour, F., Petrov, R. G., Chesneau, O., et al. 2007, A\&A, 464, 107

Moffat, A. F. J., Lamontagne, R., Williams, P. M., Horn, J., \& Seggewiss, W. 1987, ApJ, 312, 807

Monnier, J. D., Berger, J.-P., Millan-Gabet, R., et al. 2006, ApJ, 647, 444

Monnier, J. D., Traub, W. A., Schloerb, F. P., et al. 2004, ApJ, 602, L57

Morris, P. W., Brownsberger, K. R., Conti, P. S., Massey, P., \& Vacca, W. D. 1993, ApJ, 412, 324

Pauls, T. A., Young, J. S., Cotton, W. D., \& Monnier, J. D. 2005, PASP, 117, 1255

Reed, B. C. 2003, AJ, 125, 2531

Smith, L. F. 1968, MNRAS, 140, 409

Smith, L. J., Norris, R. P. F., \& Crowther, P. A. 2002, MNRAS, 337, 1309

Tannirkulam, A., Monnier, J. D., Harries, T. J., et al. 2008, ApJ, 689, 513

Taranova, O. G., \& Shenavrin, V. I. 2011, Astron. Lett., 37, 30

ten Brummelaar, T. A., McAlister, H. A., Ridgway, S. T., et al. 2005, ApJ, 628, 453

Traub, W. A., Ahearn, A., Carleton, N. P., et al. 2003, Proc. SPIE, 4838, 45

Tuthill, P. G., Monnier, J. D., Lawrance, N., et al. 2008, ApJ, 675, 698

Usov, V. V. 1991, MNRAS, 252, 49

van der Hucht, K. A. 2001, New Astron. Rev., 45, 135

White, R. L., \& Becker, R. H. 1995, ApJ, 451, 352

Williams, P. M., Marchenko, S. V., Marston, A. P., et al. 2009, MNRAS, 395 , 1749

Williams, P. M., van der Hucht, K. A., Florkowski, D. R., Pollock, A. M. T., \& Wamsteker, W. M. 1987, in IAU Symp. 122, Circumstellar Matter, ed. I. Appenzeller \& C. Jordan (Cambridge: Cambridge Univ. Press), 453

Williams, P. M., van der Hucht, K. A., Pollock, A. M. T., et al. 1990, MNRAS, 243,662

Zhao, M., Monnier, J. D., Torres, G., et al. 2007, ApJ, 659, 626 